\title{
Marco referencial de las creencias y experiencias paranormales y su relación con la esquizotipia positiva/negativa*
}

\author{
Alejandro Parra \\ Universidad Abierta Interamericana \\ Buenos Aires, Argentina
}

Recibido: 15 de abril de 2015 / Aprobado: 18 de junio de 2015

Se estudian las experiencias paranormales, percibidas de acuerdo a contextos diferenciados. Investigaciones previas demuestran el rol de las creencias paranormales o del pensamiento mágico como una influencia adaptativa y protectora potencial, tanto en la esquizotipia positiva como en la negativa. Se trabajó con 1990 participantes, 416 del grupo paranormal y 1574 no involucrados en temas paranormales; aplicándose el Inventario Oxford-Liverpool de Sentimientos y Experiencias, que evalúa cuatro dimensiones de propensión a la esquizotipia, y el Cuestionario de Experiencias Paranormales; asimismo, se diseñó un índice de experiencias paranormales según el número de respuestas afirmativas a preguntas de experiencias paranormales. Los resultados indicaron que se encontraron diferencias estadísticas significativas entre los grupos estudiados.

creencias paranormales / experiencias paranormales / esquizotipia

\section{Referential Scheme for Paranormal Beliefs and Experiences and its Relationship to Positive and Negative Schizotypes}

This study examines paranormal experiences perceived in differential contexts. Previous research shows that paranormal beliefs and/or magic though has adaptive and protective influence both in positive and negative schizotypes. The sample consisted in 1990 participants (416 paranormal group, 1574 non-paranormal group). They were tested with the Oxford-Liverpool Inventory of Feelings and Experiences (O-LIFE) which evaluates propensity to schizotype and with the Questionnaire of Paranormal Experiences. An index of paranormal experiences was designed based on the positive responses to paranormal experiences. Results show significant differences between both groups.

paranormal beliefs / paranormal experiences / schizotype

* Este estudio fue posible gracias a un subsidio otorgado por la Universidad Abierta Interamericana y a la Fundaçao BIAL de Portugal.

Correo electrónico: rapp@fiertel.com.ar 
En los últimos años se ha examinado la relación entre los rasgos de personalidad esquizotípica y las creencias paranormales. Windholz y Diamant (1974) encontraron que los creyentes en lo paranormal puntuaron significativamente más alto en la subescala de esquizofrenia del Inventario Multifásico de Personalidad de Minnesota (MMPI) (Hathaway y McKinley, 1983) en comparación con un grupo de no creyentes en lo paranormal. Thalbourne (1994) encontró que los estudiantes universitarios que creían y decían haber tenido experiencias con lo paranormal tendían a puntuar más alto en la Escala de Ideación Mágica (Eckblad y Chapman, 1983) y la Escala de Aberración Perceptual (Chapman, Chapman y Raulin, 1978). Chapman y Chapman (1987) concluyeron que los aspectos perceptuales de la esquizotipia y el pensamiento mágico son indicadores de procesos que están relacionados con la vulnerabilidad a la psicosis. Por ejemplo, Wolfradt, Oubaid, Straube, Bischoff y Mischo (1999), utilizando el Inventario de Experiencias Anómalas (Gallagher, Kumar y Pekala, 1994), y el cuestionario de personalidad esquizotípica (Raine y Benishay, 1995) encontraron que las creencias y las experiencias paranormales estaban fuertemente correlacionadas con la esquizotipia positiva.

Es notable que en el Diagnostic and Statistical Manual of Mental Disorders (DSM-IV) (American Psychiatric Association, 1994) la ideación mágica es solo uno de los nueve criterios para el diagnóstico de la esquizotipia: las creencias raras o el pensamiento mágico que influye en el comportamiento y es incompatible con las normas subculturales (por ejemplo, la superstición, la creencia en la clarividencia, la telepatía o el "sexto sentido", y fantasías y preocupaciones bizarras). (DSM-IV, 1994, p. 654)

Los ejemplos de creencias bizarras se refieren directamente a la creencia paranormal, por lo tanto, la creencia paranormal en el DSM-IV es considerada como un ejemplo de pensamiento mágico, que a su vez es una faceta del trastorno esquizotípico de personalidad. A partir de estas definiciones, la relación entre la esquizotipia y la creencia paranormal parece ser una tautología.

La esquizotipia negativa, identificada como un factor separado de la esquizotipia, y por lo general evaluada en términos de anhedonia física o social, puede ser un indicador de riesgo de perturbación mental, por ejemplo, Nettle y Clegg (2006) encontraron, en base a una muestra de artistas y poetas creativos, que la anhedonia correlacionaba negativamente con la creatividad. Los autores sostienen que es la anhedonia lo que diferencia la esquizotipia positiva de las personas creativas de las de los individuos clínicos. Personas con puntuaciones "positivas altas y negativas bajas" (y por lo tanto, en condiciones saludables) pueden canalizar sus tendencias esquizotípicas vía la creatividad, mientras que los individuos con puntuaciones "negativas altas y positivas bajas" (por 
ende, disfuncionales) sucumben a los efectos desorganizativos de la esquizotipia positiva y generan trastornos.

Parece haber gran evidencia anecdótica de un vínculo entre la creencia paranormal y una propensión a la psicosis. Partiendo del criterio de Meehl (1964), según el cual el pensamiento mágico es un síntoma de esquizotipia, esta última fue operacionalizada como "pensamiento o ideación mágica". Eckblad y Chapman (1983) construyeron la Escala de Ideación Mágica, por la cual también hay una relación con la propensión a la psicosis, similar a la esquizofrenia. Ambos definen pensamiento mágico como "creencia y plantean las experiencias como una causación no válida" (p. 215). Como algunos ítems de la Escala de Ideación Mágica incluyen elementos relacionados con los fenómenos paranormales, Tobacyk y Wilkinson (1990) investigaron la relación entre pensamiento mágico y creencias paranormales. En su estudio, el pensamiento mágico se evaluó mediante la Escala de Ideación Mágica (Eckblad y Chapman, 1983) y la creencia paranormal con la Escala de Creencias Paranormales de Tobacyk y Milford (1983).

En el contexto de las experiencias paranormales, McCreery y Claridge (2002) investigaron la relación entre las experiencias fuera del cuerpo (EFC) y diferentes dimensiones de esquizotipia (Bentall, Claridge y Slade, 1989) a personas con experiencias y sin ellas. Hubo una tendencia en personas con expe- riencia a tener menores puntuaciones de esquizotipia negativa que quienes no tuvieron la experiencia, y un mayor nivel de esquizotipia cognitivo-perceptual en aquellos que no tuvieron la experiencia. Esto no necesariamente tiene consecuencias psicopatológicas para el individuo, las personas que tienen experiencias paranormales simplemente podrían ser más proclives a tener experiencias perceptuales anómalas. Parra (2008) encontró mayor esquizotipia cognitivo-perceptual en individuos que afirman ser capaces de ver el "aura" o campo de energía que rodea a una persona en comparación con quienes no tienen esta experiencia. Posiblemente, estas personas tengan una vida imaginativa mucho más intensa. Parra y Espinoza (2010) también encontraron mayor esquizotipia cognitivo-perceptual y propensión a la alucinación en personas que decían tener experiencias extrasensoriales. Estos hallazgos sugieren que hay otros procesos disociativos subyacentes, como la absorción y propensidad a la fantasía, que se asocian con tales experiencias.

Es importante señalar que las experiencias y las creencias paranormales son conceptos diferentes, aunque ambos elementos a veces se superponen en las escalas (Eckblad y Chapman, 1983; Mason, Claridge y Jackson, 1995). Las experiencias paranormales podrían tener una función adaptativa, e incluso "protectora". Lange y Houran (1997) encontraron una reducción de la ansiedad ante la muerte en participantes con experiencias paranormales, mientras 
que Kennedy, Kanthamami y Palmer (1994) encontraron que las experiencias (pero no las creencias) paranormales estaban asociadas a un mayor sentido del significado en la vida. Kennedy y Kanthamami (1995) también encontraron relaciones entre las experiencias paranormales, con optimismo y bienestar psicológico. Williams e Irwin (1991) sugirieron que las creencias paranormales representan una defensa cognitiva contra la incertidumbre, mientras que para otros están relacionadas con la psicopatología, especialmente la esquizotipia.

Por lo tanto, un posible mecanismo para este modelo radica en la cualidad subjetiva de las experiencias paranormales. Tales experiencias encajan en un marco de creencias que se consideran más agradables, mientras que las personas sin dicho contexto las encuentran intrusivas y perturbadoras. Parra y Espinoza (2010) encontraron una diferencia significativa en síntomas positivos de esquizotipia en el grupo de estudiantes "espirituales" y "no-espirituales" ( $p=0.02)$, pero no significativo para los síntomas negativos. También se observó que todas las experiencias paranormales correlacionaban significativamente con los síntomas positivos de esquizotipia. Aunque fenómenos como la telepatía y ver el aura no se relacionan con síntomas negativos de la esquizotipia, sin embargo, se encontró una correlación significativa entre la experiencia fuera del cuerpo, la sensación de presencia y la experiencia de ver apariciones con los síntomas ne- gativos, aunque sustancialmente menor que los síntomas positivos.

Se pone a prueba la hipótesis de que aquellas personas que tienen más experiencias y creencias paranormales son capaces de hacer frente a los efectos potencialmente angustiantes de tales experiencias. Específicamente: $\left(\mathrm{H}_{1}\right)$, que el grupo paranormal puntuará más alto en experiencias inusuales, desorganización cognitiva, anhedonia introvertida, disconformidad impulsiva, la puntuación total de esquizotipia y los subfactores de esquizotipia positiva y esquizotipia negativa en comparación con el grupo no paranormal; $\left(\mathrm{H}_{2}\right)$, que el índice del grupo paranormal correlacionará positivamente con el subfactor experiencias inusuales y las puntuaciones de esquizotipia positiva/negativa; $\left(\mathrm{H}_{3}\right)$, que el índice de experiencias paranormales del grupo no paranormal (estudiantes) correlacionará positivamente con los subfactores experiencias inusuales, desorganización cognitiva, anhedonia introvertida, disconformidad impulsiva, la puntuación total de esquizotipia y los subfactores de esquizotipia positiva y esquizotipia negativa, y $\left(\mathrm{H}_{4}\right)$, que el índice del grupo no paranormal (estudiantes) correlacionaría positivamente con el grupo de experiencias inusuales, desorganización cognitiva, anhedonia introvertida, disconformidad impulsiva, la puntuación total del O-LIFE y las puntuaciones de esquizotipia positiva/negativa. 


\section{Método}

\section{Participantes}

Grupo paranormal. La muestra estuvo conformada por 416 participantes de ambos sexos, 309 (74 \%) mujeres y 107 (26\%) varones, cuyo rango etario fue de 17 a 83 años (Media=44,29; DT=13,64), reclutados en los cursos y talleres del Instituto de Psicología Paranormal de Buenos Aires, y creyentes en una variedad de experiencias vinculadas a lo paranormal. Los participantes fueron reclutados a través de anuncios en medios de prensa y una lista de correos electrónicos y en una página web (www. alipsi.com.ar). El anuncio tuvo una breve explicación del procedimiento y animó a los participantes a concertar una entrevista con nosotros para obtener más información.

Grupo no paranormal. De un total de 1850 estudiantes universitarios reclutados en el Departamento de Psicología, se recibieron 1574 cuestionarios completos $(85 \%)$. Los participantes eran adultos, en su mayoría estudiantes, en el Campus Sur de la Universidad Abierta Interamericana de la zona sur de Buenos Aires, Argentina. Se colectaron cuestionarios que incluyeron ambos sexos, 909 (57 \%) de mujeres y 665 (43\%) de varones, cuyas edades oscilan entre los 15 a 83 años (Media=33,84; $\mathrm{DE}=12,84$ ). El set de las escalas se entregó en un sobre. Cada participante, después de recibir información acerca de los objetivos del estudio (pero no las hipótesis), fue invitado a completar las escalas en forma anónima. Cuestionarios devueltos fueron almacenados sin examinar a través de la contratación y periodos de recogida. No se tenía conocimiento de los resultados hasta que el último cuestionario completado fue devuelto, en diciembre de 2011. Reconozco que la muestra no puede ser considerada como representativa y que la forma no sistemática de selección de los participantes puede haber sesgado mis resultados. Se invitó a cada participante a completar ambos cuestionarios en forma anónima, junto a un consentimiento informado.

\section{Instrumentos}

Inventario de los Sentimientos y Experiencias Oxford-Liverpool (O-LIFE) (Mason et al., 1995). Se trata de un cuestionario de 150 ítems que evalúa esquizotipia en cuatro dimensiones: esquizotipia positiva evalúa experiencias inusuales (por ejemplo, "¿Son tus pensamientos a veces tan fuertes que casi se pueden oír?") y desorganización cognitiva (por ejemplo, “¿Le resulta difícil mantener el interés en la misma cosa durante mucho tiempo?"); esquizotipia negativa evalúa dos factores: anhedonia introvertida (por ejemplo, "¿Has tenido muy poco de diversión de las actividades físicas como caminar, nadar o hacer deportes?") y disconformidad impulsiva (por ejemplo, “¿Usted se considera ser más o menos un tipo promedio de persona?"). La subescala de experiencias inusuales contiene 30 
ítems, que describen aberraciones perceptuales, pensamiento mágico y alucinaciones. Está fenomenológicamente relacionada con los síntomas positivos de la psicosis, y mide una característica conocida como "esquizotipia positiva". La subescala desorganización cognitiva contiene 24 ítems, relacionados con la capacidad de atención y concentración, pobre capacidad para la toma de decisiones y ansiedad social. La subescala anhedonia introvertida contiene 27 ítems, que describen la falta de placer en las relaciones sociales, y evitación de la intimidad. La subescala de disconformidad impulsiva contiene 23 ítems, que describen impulsividad, conductas antisociales y comportamiento excéntrico, que a veces sugiere falta de autocontrol. La evaluación psicométrica de la escala ha mostrado tener buena fiabilidad test-retest (coeficiente alpha $=.80$ ), así como una aceptable consistencia interna (alfa Cronbach=.77) (Mason et al., 1995; Mason, Claridge y Claridge, 1995). La medida de consistencia interna sobre la población argentina fue de .91 .

Encuesta de experiencias paranormales. Hemos desarrollado un inventario autoadministrado para recolectar información sobre experiencias paranormales espontáneas (Gómez y Parra, 2008). Se trata de un cuestionario de 10 ítems, que incluye experiencias subjetivas, como sueños premonitorios, telepatía, ver aura, experiencias fuera del cuerpo, sensación de presencia, mediumnidad, experiencia de sanación (como sanador), déjà-vu, experiencia mística y apariciones (oír o ver fantasmas), que el participante responde como "Nunca", "Rara vez" o "Múltiples veces".

Se construyó un índice o recuento de experiencias paranormales para cada participante en función del número de respuestas afirmativas a sus experiencias paranormales. Este índice tenía un rango de 0 (ninguna experiencia) al 10 (todas las experiencias). El índice también fue correlacionado con los factores del O-LIFE.

\section{Análisis de datos}

Los datos fueron procesados mediante el paquete estadístico SPSS 20. Se llevó a cabo una evaluación de la normalidad de la muestra. A partir de los valores obtenidos a través del estadístico ShapiroWilks, se asumió una distribución asimétrica de la puntuación de ambos instrumentos. En consecuencia, se empleó estadísticas no paramétricas. Para comparar ambas muestras se empleó el análisis de $U$ de Mann-Whittney, y para calcular las correlaciones se aplicó el coeficiente Rho de Spearman y se utilizó $\mathrm{r}$ como estimación del tamaño del efecto.

\section{Resultados}

En el grupo "paranormal" las experiencias más frecuentes son sanación (80 \%), sueños premonitorios (79 \%) y telepatía $(68,5 \%)$, en cambio para el grupo no-paranormal las experiencias más frecuentes son sueños premonitorios 
(40 \%), sensación de presencia (39,6\%), y telepatía $(38,6 \%)$.

El análisis comparativo de los datos se realizó empleando el test $U$ de MannWhittney entre los participantes que reportan experiencias paranormales y quienes no las reportan, los hallazgos indicaron que se encontraron diferencias estadísticas significativas entre ambos grupos en todos los casos $(\mathrm{p}<.001)$, notándose que los tamaños del efecto fluctuaron entre pequeño $(\mathrm{r}=.12)$ y Mediano $(\mathrm{r}=.43)$.

Con respecto a los contrastes de hipótesis los resultados indicaron lo siguiente:

- La hipótesis H1 plantea que el grupo paranormal puntuaría más alto en experiencias inusuales, desorganización cognitiva, anhedonia introvertida, disconformidad impulsiva, las puntuaciones totales $\mathrm{y}$ las puntuaciones de esquizotipia

Tabla 1

Frecuencia y porcentaje de experiencias paranormales en ambos grupos

\begin{tabular}{|c|c|c|c|}
\hline $\begin{array}{l}\text { Experiencias } \\
\text { paranormales }\end{array}$ & $\begin{array}{c}\text { Grupo } \\
\text { paranormal } \\
(N=416)\end{array}$ & $\begin{array}{l}\text { Experiencias } \\
\text { paranormales }\end{array}$ & $\begin{array}{c}\text { Grupo } \\
\text { no-paranormal } \\
(N=1574)\end{array}$ \\
\hline Sanación & $333(80,0 \%)$ & $\begin{array}{l}\text { Sueños } \\
\text { premonitorios }\end{array}$ & $628(40,0 \%)$ \\
\hline Sueños premonitorios & $331(79,6 \%)$ & $\begin{array}{l}\text { Sensación de } \\
\text { presencia }\end{array}$ & $623(39,6 \%)$ \\
\hline Telepatía & $285(68,5 \%)$ & Telepatía & $608(38,6 \%)$ \\
\hline Psicokinesis espontánea & $256(61,5 \%)$ & Sanación & $322(20,5 \%)$ \\
\hline Sensación de presencia & $240(57,7 \%)$ & $\begin{array}{l}\text { Experiencias } \\
\text { extracorporales }\end{array}$ & $288(18,3 \%)$ \\
\hline Experiencia mística & $223(53,6 \%)$ & $\begin{array}{l}\text { Psicokinesis } \\
\text { espontánea }\end{array}$ & $273(17,3 \%)$ \\
\hline Mediumnidad & $211(50,7 \%)$ & Ver el aura & $236(15,0 \%)$ \\
\hline $\begin{array}{l}\text { Experiencias } \\
\text { extracorporales }\end{array}$ & $191(45,9 \%)$ & $\begin{array}{l}\text { Experiencia } \\
\text { mística }\end{array}$ & $226(14,3 \%)$ \\
\hline Apariciones & $147(35,3 \%)$ & Apariciones & $186(11,8 \%)$ \\
\hline Ver el aura & $127(30,5 \%)$ & Mediumnidad & $172(10,9 \%)$ \\
\hline
\end{tabular}


positiva/negativa en comparación con el grupo no paranormal, la cual se confirmó: en la medida en que las medias fueron significativamente mayores en el grupo paranormal en comparación con el grupo no paranormal $(\mathrm{p}<.001$, a una cola) (véase la tabla 2).

- En relación a la hipótesis H2, que indica que el grupo paranormal puntuaría más alto en el IEP (recuento de experiencias) en comparación con el grupo no paranormal, la cual fue confirmada. La media de experiencias $(6,36)$ del grupo paranormal fue significativamente más alta que el grupo no paranormal $(3,00)(\mathrm{p}<.001$, una cola) (véase la tabla 2).

- En lo concerniente a la hipótesis H3, la cual indica que el IEP del grupo paranormal correlacionará positivamente con los factores experiencias inusuales y las puntuaciones de esquizotipia positiva y negativa, lo cual solo se confirmó para experiencias inusuales $(\mathrm{r} s=10)$. En sentido inverso, el

Tabla 2

Comparación de los puntajes del O-LIFE y el índice de experiencias paranormales, entre el grupo paranormal y no-paranormal

\begin{tabular}{|c|c|c|c|c|c|c|c|}
\hline \multirow[b]{3}{*}{ O-life } & \multicolumn{4}{|c|}{ Grupo ${ }^{(a)}$} & \multirow{3}{*}{$\begin{array}{c}U d e \\
\text { Mann- } \\
\text { Whittney }\end{array}$} & \multirow[b]{3}{*}{$z$} & \multirow[b]{3}{*}{$r$} \\
\hline & \multicolumn{2}{|c|}{ Paranormal } & \multicolumn{2}{|c|}{$\begin{array}{c}\text { No } \\
\text { paranormal }\end{array}$} & & & \\
\hline & $M$ & $D E$ & $M$ & $D E$ & & & \\
\hline $\begin{array}{l}\text { 1. Experiencias } \\
\text { inusuales (EI) }\end{array}$ & 0.73 & 1.12 & 0.34 & 0.24 & 177361 & $14.40 * * *$ & 0.32 \\
\hline $\begin{array}{l}\text { 2. Desorganización } \\
\text { cognitiva (DC) }\end{array}$ & 0.78 & 1.28 & 0.42 & 0.23 & 265954 & $5.90 * * *$ & 0.13 \\
\hline $\begin{array}{l}\text { 3. Anhedonia } \\
\text { introvertida (AI) }\end{array}$ & 0.87 & 0.92 & 0.34 & 0.23 & 134072 & $18.56 * * *$ & 0.42 \\
\hline $\begin{array}{l}\text { 4. Desconformidad } \\
\text { impulsiva (DI) }\end{array}$ & 0.46 & 0.55 & 0.29 & 0.20 & 269737 & $5.53^{* * *}$ & 0.12 \\
\hline $\begin{array}{l}\text { F1. Esquizotipia } \\
\text { positiva }(E I+D C)\end{array}$ & 1.51 & 2.33 & 0.76 & 0.40 & 202442 & $11.54 * * *$ & 0.26 \\
\hline $\begin{array}{l}\text { F2. Esquizotipia } \\
\text { negativa }(I A+I N)\end{array}$ & 1.34 & 1.41 & 0.63 & 0.37 & 155158 & $15.04 * * *$ & 0.34 \\
\hline $\begin{array}{l}\text { Esquizotipia } \\
\text { (Puntaje total) }\end{array}$ & 2.87 & 3.64 & 1.39 & 0.63 & 169635 & $14.23 * * *$ & 0.32 \\
\hline
\end{tabular}

Nota: (a) debido a que los datos no tenían una distribución asimétrica, se empleó el factor de correlación Rho de Spearman para testear las hipótesis: ${ }^{*} \mathrm{p}<.05$; $*$ * $\mathrm{p}<.01 ;{ }^{* * *} \mathrm{p}<.001$ (todos los valores de $p$ a dos colas). Se aplicó $r$ como medida de tamaño del efecto. 
Tabla 3

Correlaciones entre esquizotipia y el índice de experiencias(a)

\begin{tabular}{|c|c|c|c|c|c|c|c|}
\hline Index ${ }^{(\mathrm{b})}$ & El & $D C$ & $A /$ & $D I$ & Esquizotipia & $\begin{array}{c}\text { Esquizotipia } \\
\text { positiva }\end{array}$ & $\begin{array}{c}\text { Esquizotipic } \\
\text { negativa }\end{array}$ \\
\hline $\begin{array}{l}\text { Grupo } \\
\text { paranormal }\end{array}$ & $.10^{*}$ & $-.13^{*}$ & .06 & .03 & .03 & .04 & .02 \\
\hline $\begin{array}{l}\text { Grupo no } \\
\text { paranormal }\end{array}$ & $.28 * *$ & $.05^{*}$ & .06 & $-.13^{* *}$ & $.11^{* *}$ & $.19 * *$ & -.02 \\
\hline
\end{tabular}

Nota: (a) Debido a que los datos no tenían una distribución asimétrica, se empleó el factor de correlación Rho de Spearman para testear las hipótesis: ${ }^{*} p<.05$; ${ }^{* *} p<.01$; ${ }^{* *} p<.001$ ( $p$ a dos colas). (b) Siendo $0=$ Sin experiencias a $10=$ Diez experiencias.

IEP correlacionó negativamente con desorganización cognitiva ( $r s=-.13)$ (véase la tabla 3).

- H4 plantea que el índice del grupo no paranormal (estudiantes) correlacionaría positivamente con el grupo de experiencias inusuales, desorganización cognitiva, anhedonia introvertida, disconformidad impulsiva, la puntuación total del O-LIFE y las puntuaciones de esquizotipia positiva/negativa, las cuales se confirmaron pero solo para experiencias inusuales ( $\mathrm{rs}=.28)$, desorganización cognitiva $(\mathrm{r} s=.05)$, anhedonia introvertida $(\mathrm{r} s=.06)$, Total O-LIFE $(\mathrm{r} s=11)$, y esquizotipia positiva $(\mathrm{r} s=19)$. En el sentido inverso, el index correlacionó negativamente en disconformidad impulsiva $(\mathrm{r} s=-.13)$ (véase la tabla 3$)$.

\section{DisCusión}

Los análisis revelaron una relación entre esquizotipia positiva y experiencias paranormales para el grupo no paranormal pero ninguna correlación para el grupo paranormal en línea con las predicciones; en cambio el grupo con experiencias paranormales (o con un contexto de creencias) era cognitivamente menos desorganizado. La esquizotipia positiva -pero no la negativa- correlacionó con las experiencias paranormales, también en línea con las predicciones, asociando experiencias paranormales con alta anhedonia en el grupo paranormal.

El grupo paranormal resultó cognitivamente menos desorganizado y tiende a tener más experiencias inusuales en relación con sus experiencias paranormales. Las personas que tienen más experiencias y creencias paranormales 
son capaces de hacer frente a los efectos potencialmente angustiantes de tales experiencias.

Esto se integra bien con un estudio realizado por Williams e Irwin (1991), en el que se encontró que el estilo cognitivo difería entre participantes que puntuaban alto en esquizotipia positiva, y aquellos que no puntuaban pero tenían creencias paranormales. Los autores sugieren que tener un contexto de creencias puede actuar como un mecanismo de protección, permitiendo a los individuos aceptar y explicar sus experiencias paranormales, mientras que los que no tienen tal contexto serán "bombardeados" por sucesos extraños para los que no tienen explicación. Por lo tanto, los participantes que tienen mayor desorganización cognitiva probablemente tienen menos protección y estarían en mayor riesgo de padecer angustia y disfunción.

Para el grupo paranormal, las personas que tienen más experiencias inusuales pueden "amortiguar" los efectos potencialmente angustiantes de las experiencias paranormales a través de la existencia o la construcción de un contexto donde colocarlos; para el grupo no paranormal (individuos sin creencias), la esquizotipia positiva puede, de hecho, ser adaptativa y el pensamiento mágico permite construir un marco referencial más eficaz e imaginativo, que da cuenta de las experiencias extrañas, como sugieren los resultados de Bell, Halligan y Ellis (2006). Para estas personas, la falta de un "amortiguador" da lugar a experiencias que son vistas como extrañas y abrumadoras, o quizás indicativas de algún trastorno o "anormalidad".

Una limitación del presente estudio es que no examinó las creencias paranormales de los participantes. No obstante, en otros estudios (Parra, 2010, 2011, 2012a, 2012b) se observó que las creencias y las experiencias paranormales están fuertemente correlacionadas (para una revisión de estudios y metaanálisis, véase Irwin, 2009). La muestra de individuos del grupo no paranormal estaba integrada por una amplia gama de estudiantes, lo cual pudo haber sesgado la muestra. Debemos ser cautos para interpretar los resultados. No obstante, futuros estudios pueden mejorar el diseño y ayudar a comprender mejor la relación entre tales variables. En conclusión, los resultados indican un potencial adaptativo y un efecto protector, de las creencias paranormales y el pensamiento mágico para el grupo paranormal; la calidad de las creencias paranormales como adaptativas pueden ser influidas por la esquizotipia, tanto positiva como negativa, una relación moderada con el grado de desorganización cognitiva del participante.

\section{REFERENCIAS}

American Psychiatric Association. (1994). Diagnostic and statistical manual of mental disorders, DSM-IV. Washington, DC: American Psychiatric Association. 
Bell, V., Halligan, P. W., y Ellis, H. D. (2006). The cardiff anomalous perceptions scale (CAPS): A new validated measure of anomalous experience. Schizophrenia Bulletin, 32, 366-377.

Bentall, R. P., Claridge, G. S., y Slade, P. D. (1989). The multidimensional nature of schizotypal traits: A factor analytic study with normal subjects. British Journal of Clinical Psychology, 28, 363-375.

Chapman, L. J., y Chapman, J. P. (1987). Psychosis proneness. En M. Alpert (Ed.), Controversies in Schizophrenia. New York: Guilford Press.

Chapman, L. J., Chapman, T. P., y Raulin, M. C. (1978). Body-image aberration in schizophrenia. Journal of Abnormal Psychology, 87, 399-407.

Claridge, G. (Ed.). (1997). Schizotypy: Implications for illness and health. Oxford: Oxford University Press.

Eckblad, M., y Chapman, L.J. (1983). Magical ideation as an indicator of schizotypy. Journal of Consulting and Clinical Psychology, 51, 215-225.

Gallagher, C., Kumar, V. K., y Pekala, R. J. (1994). The Anomalous Experiences Inventory: reliability and validity. Journal of Parapsychology, 58, 402-428.

Gómez Montanelli, A., y Parra, A. (2008). Are spontaneous anomalous/ paranormal experiences disturbing?: A survey among under-graduate students. International Journal of Parapsychology, 13, 1-14.

Hathaway, S. R., y McKinley, J. C. (1983). Minnerota Multiphasic Personality Inventory. Manual for administration and scoring. Minneapolis: University of Minnesota Press.

Irwin, H. J. (2009). The psychology of paranormal belief: A researcher's handbook. Hertfordshire, UK: University of Hertfordshire Press.

Kennedy, J. E., y Kanthamami, H. (1995). An exploratory study of the effects of paranormal and spiritual experience on peoples' lives and well-being. Journal of the American Society for Psychical Research, 89, 249-264.

Kennedy, J. E., Kanthamami, H., y Palmer, J. (1994). Psychic and spiritual experiences, health, wellbeing, and meaning in life. Journal of Parapsychology, 58, 353-383.

Lange, R., y Houran, J. (1997). Death anxiety and the paranormal: The primacy of belief over experience. Journal of Nervous and Mental Disease, 185, 584-586.

Mason, O., Claridge, G., y Jackson, M. (1995). New scales for the assessment of schizotypy. Personality and Individual Differences, 18, 7-13.

Mason, O., Claridge, G., y Williams, L. (1997). Questionnaire measurement. En G. Claridge (Ed.), Schizotypy: Implications for Illness and Health (pp. 19-37). Oxford: Oxford University Press. 
McCreery, C., y Claridge, G. (1995). Outof-body experiences and personality. Journal of the Society for Psychical Research, 60, 129-148.

Meehl, P. E., (1964). Schizotaxia, schizotypy, schizophrenia. American Psychologist. 17, 827-838.

Nettle, D., y Clegg, H. (2006). Schizotypy, creativity and mating success in humans. Proceedings of the Royal Society of London, Series B: Biological Sciences, 273, 611-615.

Parra, A. (2008). Efectos de las experiencias espirituales/paranormales en la vida de las personas y su bienestar psicológico. Revista Argentina de Clínica Psicológica, 17, 233-242.

Parra, A. (2010). Experiencias extrasensoriales y experiencias alucinatorias: Examinando la hipótesis del continuo de experiencias esquizotípicas. Liberabit, 16, 1-10.

Parra, A., y Espinoza Paul, L. (2010). Comparación entre la esquizotipia positiva y perturbadora con la espiritualidad y las experiencias paranormales en población no-clínica. Revista Argentina de Clínica Psicológica, 19, 163-172.

Parra, A. (2011). Indicadores de propensión a la esquizotipia en individuos creyentes en lo paranormal: examinando la intensidad de la imaginería y las experiencias alucinatorias. Psicología: Teoria e Prática, 12, 78-94.

Parra, A. (2012a). Relación entre las experiencias paranormales y esqui- zotipia positiva/negativa. Acta Psiquiátrica y Psicológica de América Latina, 58(4), 246-255.

Parra, A. (2012b). Experiencias perceptuales inusuales, experiencias anómalo/paranormales y propensión a la esquizotipia. Universitas Psychologica, 11(1), 657-666.

Raine, A., y Benishay, D. (1995). The SPQ-B: a brief screening instrument for schizotypal personality disorder. Journal of Personal. Disorder. 9, 346-355.

Thalbourne, M. A. (1994). Belief in the paranortnal and its relationship to schizophrenia-relevant measures: a confixmatory study. British Journal of Clinical Psychology, 33, 78-80.

Tobacyk, J., y Milford, G. (1983). Belief in paranormal phenomena: Assessment instrument development and implications for personality functioning. Journal of Personality and Social Psychology, 44, 1029-1037.

Tobacyk, J., y Wilkinson, L. V. (1990). Magical thinking and paranormal beliefs. Journal of Social Behavior and Personality, 5, 255-264.

Williams, L. M., e Irwin, H. J. (1991). A study of paranormal belief, magical ideation as an index of schizotypy, and cognitive style. Personality and Individual Differences, 12, 1339-1348.

Windholz, G., y Diamont, L. (1974). Some personality traits of believers in ex- 
traordinary phenomena. Bulletin of the Psychonomic Society, 3, 125-126.

Wolfradt, U., Oubaid, V., Straube, E. R., Bischoff, N., y Mischo, J. (1999).
Thinking styles, schizotypal traits and anomalous experiences. Personality and Individual Differences, 27, 821-830. 
